\title{
Leishmania Parasites Differently Regulate Antioxidant Genes in Macrophages Derived From Resistant and Susceptible Mice
}

\author{
Haifa Bichiou ${ }^{1,2}$, Sameh Rabhi ${ }^{1}$, Cherif Ben Hamda ${ }^{1}$, Cyrine Bouabid ${ }^{1,2}$, Meriam Belghith $^{3}$, \\ David Piquemal ${ }^{4}$, Bernadette Trentin ${ }^{4}$, Imen Rabhi ${ }^{1,5}$ and Lamia Guizani-Tabbane ${ }^{1 *}$ \\ ${ }^{1}$ Laboratory of Medical Parasitology, Biotechnology and Biomolecules, Institut Pasteur de Tunis, Tunis-Belvedere, Tunisia, \\ ${ }^{2}$ Faculty of Sciences of Tunis, Université de Tunis El Manar, Tunis, Tunisia, ${ }^{3}$ Department of Immunology, Institut Pasteur de \\ Tunis, University Tunis El-Manar, Tunis, Tunisia, ${ }^{4}$ Acobiom, Grabels, France, ${ }^{5}$ Higher Institute of Biotechnology at Sidi- \\ Thabet, Biotechpole Sidi-Thabet, University of Manouba, Sidi-Thabet, Tunisia
}

OPEN ACCESS

Edited by:

Maria E. Francia,

Institut Pasteur de Montevideo,

Uruguay

Reviewed by:

Carlos Robello,

Universidad de la República, Uruguay

Lesly Temesvari,

Clemson University, United States

*Correspondence:

Lamia Guizani-Tabbane lamia.guizani@pasteur.rns.tn

Specialty section:

This article was submitted to

Parasite and Host,

a section of the journal

Frontiers in Cellular and Infection

Microbiology

Received: 28 July 2021 Accepted: 20 September 2021

Published: 15 October 2021

Citation:

Bichiou H, Rabhi S,

Ben Hamda C, Bouabid C

Belghith M, Piquemal D, Trentin B,

Rabhi I and Guizani-Tabbane L (2021)

Leishmania Parasites Differently

Regulate Antioxidant Genes in

Macrophages Derived From

Resistant and Susceptible Mice.

Front. Cell. Infect. Microbiol. 11:748738.

doi: 10.3389/fcimb.2021.748738
Macrophage-Leishmania interactions are central to parasite growth and disease outcome. Macrophages have developed various strategies to fight invaders, including oxidative burst. While some microorganisms seem to survive and even thrive in an oxidative environment, others are susceptible and get killed. To counter oxidative stress, macrophages switch the expressions of cytoprotective and detoxifying enzymes, which are downstream targets of the nuclear factor erythroid 2-related factor 2 (Nrf2), to enhance cell survival. We have explored the transcription of NRF2 and of its target genes and compared the effect of the parasite on their transcription in bone marrow-derived macrophages (BMdMs) from Leishmania-resistant and Leishmaniasusceptible mice. While heme oxygenase $1(\mathrm{HO}-1)$ transcription is independent of the genetic background, the transcription of glutathione reductase (Gsr) and of cysteine/ glutamate exchange transporter (Slc7a11), involved in glutathione accumulation, was differentially regulated in BMdMs from both mouse strains. We also show that, except for $\mathrm{HO}-1$, known to favor the survival of the parasite, the transcription of the selected genes, including Gsr, CD36, and catalase (CAT), was actively repressed, if not at all time points at least at the later ones, by the parasite, especially in Balb/c BMdMs. Consistent with these results, we found that the silencing of NRF2 in this study increases the survival and multiplication of the parasite.

Keywords: Leishmania, macrophage, susceptibility, resistance, NRF2, antioxidants

\section{INTRODUCTION}

Leishmaniasis is a parasitic disease caused by the protozoa Leishmania. This disease is spread worldwide and causes different clinical manifestations ranging from cutaneous lesions healing spontaneously to visceral leishmaniasis, which is the most serious form of the disease that is fatal in the absence of treatment. Leishmania parasites alternate between two life stages, and the flagellated promastigotes 
injected by the female sand fly transform into amastigotes when infecting host immune cells. As a main host for Leishmania replication, macrophages play a critical role in the outcome of the disease and in the battle between these two players: the host fighting the invaders with all available arsenal and the parasites developing various strategies to subvert the microbicidal functions of their host cells (Conceição-Silva and Morgado, 2019). Indeed, macrophages encounter Leishmania by employing an array of directly antimicrobial mechanisms such as the generation of reactive oxygen species (ROS) and reactive nitrogen species (RNS), which are highly destructive to Leishmania.

These oxidant compounds, while crucial in clearing invading pathogens, may cause oxidative damage to the host cells. Cells have developed different antioxidant defense systems to counter oxidative stress. These include the thioredoxin (Trx) and glutathione (GSH) systems, the two major thiol-dependent antioxidant mechanisms in cells, and heme oxygenase 1 (HO1), which catalyzes the rate-limiting step of heme oxidation to biliverdin, carbon monoxide, and free ferrous iron (Tonelli et al., 2018). Most of these cytoprotective and detoxifying enzymes that enhance cell survival are downstream targets of the nuclear factor erythroid 2-related factor 2 (Nrf2).

$\mathrm{Nrf} 2$ is a stress-responsive transcription factor encoded by the NFE2L2 gene in humans and is a member of the CNC ("cap ' $\mathrm{n}$ ' collar") subfamily of basic region leucine zipper (bZIP) transcription factors (Sykiotis and Bohmann, 2010). During homeostasis, Nrf2 is sequestered in the cytoplasm by Kelch-like ECH-associated protein 1 (Keap1, Nrf2 repressor protein), which drives NRF2 to ubiquitindependent proteasomal degradation (Baird and Yamamoto, 2020). After redox perturbation, Nrf2 is released from Keap1 and translocates into the nucleus. It then forms heterodimers with small Maf proteins and binds antioxidant response elements (AREs) regulating a broad range of expressions of antioxidant and detoxification genes (Itoh et al., 1999). The promoter of the Nrf2 gene itself contains AREs and amplifies the redox cascades via positive feedback regulation (Nguyen et al., 2003).

To clarify the contribution of the Nrf2/HO- 1 axis and that of other detoxifying enzymes during Leishmania major infection, we explored the activation of Nrf2 and the transcription of target genes in murine macrophages from mice resistant or susceptible to Leishmania parasite infection.

\section{MATERIALS AND METHODS}

\section{Ethics Statement}

All mouse work was done according to the directive 86/609/EEC of the European Parliament and of the Council on the Protection of Animals Used for Scientific Purposes. Approval for mouse experiments was obtained from the Ethics Committee of Institute Pasteur of Tunis, with ethics approval no. 1204.

\section{Parasites and Cell Culture}

L. major (MHOM/TN/95/GLC94 zymodeme MON25) were maintained at $26^{\circ} \mathrm{C}$ in RPMI 1640 medium (Sigma, Taufkirchen, Germany) supplemented with $10 \%$ heat-inactivated fetal calf serum (Gibco, Waltham, MA, USA), penicillin (200 U/ml), streptomycin
$(200 \mu \mathrm{g} / \mathrm{ml})$, and glutamine $(4 \mathrm{mM})$. In all assays, promastigotes in the stationary phase of growth (5-6 days) were washed in culture medium at 3,000 rpm for $10 \mathrm{~min}$ at $22^{\circ} \mathrm{C}$, resuspended in medium again, and used for infection. Parasites are killed by a 10-min incubation at $100^{\circ} \mathrm{C}$. Ds-Red promastigotes were generated as previously described (Rabhi et al., 2016). Parasites were maintained in culture in medium containing $25 \mu \mathrm{g} / \mathrm{ml}$ hygromycin $\mathrm{B}$.

The mouse macrophage leukemia cell line Raw264.7 (ATCC, Manassas, VA, USA) was maintained in RPMI 1640 medium (Sigma) supplemented with $10 \%$ fetal bovine serum (FBS; Gibco), penicillin $(200 \mathrm{U} / \mathrm{ml})$, streptomycin $(200 \mu \mathrm{g} / \mathrm{ml})$, and glutamine ( $4 \mathrm{mM}$; SigmaG1146) at $37^{\circ} \mathrm{C}$ in a humidified incubator with $5 \% \mathrm{CO}_{2}$. Macrophages were cultured in 6-well plates and incubated for $12-24 \mathrm{~h}$ at $37^{\circ} \mathrm{C}$ with $5 \% \mathrm{CO}_{2}$ to adhere and then infected with Leishmania promastigotes at a ratio of 10:1. Macrophages interacting with $L$. major were maintained at $37^{\circ} \mathrm{C}$ with $5 \% \mathrm{CO}_{2}$. The infected cells were then harvested at different time points. Infections were confirmed using Giemsa staining.

$\mathrm{Balb} / \mathrm{c}$ and C57BL/6 mice (Elevage Janvier, Le Genest-SaintIsle, France) were killed and their hind legs removed for the isolation of bone marrow-derived macrophages (BMdMs). Briefly, the femurs and tibias were flushed with RPMI 1640 using a 25gauge needle. Contaminating erythrocytes were lysed through the addition of a lysis solution. All cells were incubated in T75 culture flasks at $1.5 \times 10^{6}$ cells $/ \mathrm{ml}$ in complete media added with $80 \mathrm{ng} / \mathrm{ml}$ macrophage colony-stimulating factor (M-CSF; Peprotech, Neuilly sur Seine, France) overnight for stromal cell elimination. Non-adherent, immature macrophages were transferred to fresh culture-treated Petri dishes (Nunc, Rochester, NY, USA) and grown for 7 days, with re-feeding on day 3 to induce macrophage differentiation. The purity of BMdMs was analyzed through the evaluation of the phenotypic expression of a specific macrophage subset surface marker (F4/80) by flow cytometry. Of the generated macrophages, $80 \%-90 \%$ were F4/80-positive.

\section{Knockdown Macrophage Generation}

Raw264.7 cells were seeded 18-20 h pre-transfection in 24-well plates at a confluency of $70 \%$. The cells were transfected using the ESCORTTM II transfection reagent (Sigma) with the empty plasmid non-target pLKO (NT-pLKO) or one of the five different NRF2 DNAs and extracted using Maxiprep from the NFE2L2 Mission shRNA Glycerol Stock (Sigma) according to the manufacturer's instructions.

Briefly, $2 \mu \mathrm{g}$ of DNA was diluted and incubated for $20 \mathrm{~min}$ with the diluted ESCORT II reagent at room temperature to form the ESCORT II/DNA complexes. These complexes were then added to the cells growing in serum-containing culture medium and incubated at $37^{\circ} \mathrm{C}$. At $24 \mathrm{~h}$ post-transfection, fresh growth medium was added. For selection, puromycin was added to the fresh medium $72 \mathrm{~h}$ post-transfection. The cells were kept in culture for 1 month with a cell medium change approximately two times a week. The cells were then cryopreserved after assessment of the silencing efficiency by Western blotting. Wild-type (WT), NT-pLKO, and si-Nrf2 cells were stimulated with $100 \mathrm{ng} / \mathrm{ml}$ lipopolysaccharide (LPS) and the intensities of the bands were compared. Cells transfected with the NRF2 clone, 
which gave the higher silencing percentage, and the corresponding ones transfected with the NT-pLKO plasmids were used for subsequent experiments.

\section{Ds-Red Leishmania Parasite Quantification by Flow Cytometry}

We used Ds-Red-GLC94 L. major parasites (Rabhi et al., 2016) to infect transfected si-NRF2 and NT-pLKO cells. Seventy-two hours post-infection, the cells were immediately analyzed without fixation for the estimation of parasite multiplication by flow cytometry using a Becton Dickinson FACSCanto II flow cytometer. The analysis was fulfilled with the BD FACSDiva 6 software (Becton Dickinson, Franklin Lakes, NJ, USA).

\section{Protein Extraction and Cell Fractionation}

Cells were harvested at different time points, washed gently with ice-cold phosphate-buffered saline (PBS), scraped, and centrifuged at 1,200 rpm for $10 \mathrm{~min}$. Nuclear and cytoplasmic protein extracts were prepared using a hypotonic hypertonic buffer. The pellets were resuspended in $80 \mu \mathrm{l}$ volume of hypotonic buffer containing $10 \mathrm{mM}$ HEPES-KOH ( $\mathrm{pH}$ 7.6), 60 $\mathrm{mM} \mathrm{KCl}, 1 \mathrm{mM}$ EDTA, $1 \mu \mathrm{g} / \mathrm{ml}$ aprotonin, $1 \mathrm{mM}$ orthovanadate, 1 $\mathrm{mM}$ dithiothreitol (DTT), and $1 \mathrm{mM}$ phenylmethylsulfonyl fluoride (PMSF) for isolation of the cytoplasmic extract. After incubation on ice for $10 \mathrm{~min}, 1 / 30$ on the volume of $10 \%$ NP40 was added for $1 \mathrm{~min}$ and the samples then centrifuged at 10,000 rpm for $5 \mathrm{~min}$. The cytoplasmic extract was collected and stored. The nuclear pellet was resuspended in hypertonic buffer containing $10 \mathrm{mM}$ HEPES-KOH (pH 7.6), $1.5 \mathrm{mM} \mathrm{MgCl}_{2}, 420$ $\mathrm{mM} \mathrm{NaCl}, 0.2 \mathrm{mM}$ EDTA, 25\% glycerol, $1 \mu \mathrm{g} / \mathrm{ml}$ aprotonin, $1 \mathrm{mM}$ orthovanadate, $1 \mathrm{mM}$ DTT, and $1 \mathrm{mM}$ PMSF. The nuclear pellets in hypotonic buffer were vortexed to homogenize the lysate, sonicated using Vibra-Cell at amplitude of $60 \%$ for three cycles of $10 \mathrm{~s}$ with 10-s pauses while being kept, and incubated on ice for $30 \mathrm{~min}$. The lysates were then centrifuged at $14,000 \mathrm{rpm}$ for 20 min to pellet any cell debris and the supernatants containing the nuclear proteins were collected into new tubes. For whole cell lysis, we used $30 \mu \mathrm{l}$ of lysis buffer containing $10 \mathrm{mM}$ Tris- $\mathrm{HCl}$ ( $\mathrm{pH} 7.5$ ), $50 \mathrm{mM} \mathrm{NaCl}, 50 \mathrm{mM}$ sodium fluoride (NaF), 2 mM EDTA, $1 \mathrm{mM}$ EGTA, 2\% Nonidet P-40 (NP-40), 0.75\% sodium deoxycholate (DOC), $1 \mathrm{mM}$ orthovanadate, $1 \mu \mathrm{g} / \mathrm{ml}$ aprotinin, $1 \mathrm{mM}$ PMSF, and $1 \mathrm{mM}$ DTT. After $10 \mathrm{~min}$ incubation on ice, the extracts were centrifuged at 15,000 rpm for $20 \mathrm{~min}$. All extracts were stored at $-20^{\circ} \mathrm{C}$. The protein content of each compartment was determined using the bicinchoninic acid (BCA) protein assay (Sigma).

\section{Western Blotting}

Equal amounts of protein were separated by $10 \%$ sodium dodecyl sulfate polyacrylamide gel electrophoresis and transferred onto a $0.45-\mu \mathrm{m}$ polyvinylidene difluoride (PVDF) immunoblot membrane (Amersham, Amersham, UK). After membrane blocking with 5\% skimmed milk in PBS with $0.01 \%$ Tween 20 (wash buffer) for $1 \mathrm{~h}$ at room temperature (RT), the membranes were incubated overnight at $4^{\circ} \mathrm{C}$ with commercial primary antibodies against phospho-AKT (Cell Signaling, Danvers, MA, USA), HO-1 (Santa Cruz Biotechnology, Dallas, TX, USA), NRF2 (Santa Cruz Biotechnology), histone (Cell Signaling), $\beta$-actin
(Sigma-Aldrich, St Louis, MO, USA), and PARP (Cell Signaling). The next day, the membranes were washed three times and then incubated with horseradish peroxidase-conjugated anti-rabbit secondary antibody (1:2,000; Dako, Carpinteria, CA, USA) for another $1 \mathrm{~h}$ at RT. Blots were visualized with a densitometric ECL kit (Amersham) and analysis was performed using ImageJ software.

To analyze the effects of kinase inhibitors on the expressions of Nrf2 proteins, seeded raw cells in dishes were pretreated with wortmannin $(200 \mathrm{mM})$ for $3 \mathrm{~h}$; then, the cells were washed and infected with the Leishmania promastigotes for different times. The cell lysates prepared using the lysis buffer, as described above, were subjected to Western blot analysis.

\section{RNA Extraction and qRT-PCR}

Total RNA from macrophages was isolated using the TRIzol reagent (Sigma). Total RNAs from uninfected and infected macrophages were prepared using the RNeasy mini kit (Qiagen, Hilden, Germany). RNA quantity was controlled using the NanoDrop ND-1000 micro-spectrophotometer, and RNA quality and integrity (RNA integrity no. RIN.9) were monitored on the Agilent RNA Pico LabChips (Agilent Technologies, Palo Alto, CA, USA). Reverse transcriptions were performed for each sample in a final reaction volume of $20 \mu \mathrm{l}$ with $273 \mathrm{ng}$ of total RNA using $200 \mathrm{U}$ of SuperScript III enzyme (M-MLV Reverse Transcriptase, Invitrogen) and $250 \mathrm{ng}$ of random primers according to the manufacturer's instructions $\left(25^{\circ} \mathrm{C}\right.$ for $10 \mathrm{~min}, 42^{\circ} \mathrm{C}$ for 50 $\mathrm{min}$, and $70^{\circ} \mathrm{C}$ for $15 \mathrm{~min}$ ). A negative control was included by performing reverse transcription with no template. Quantitative PCR (qPCR) experiments were carried out using EVA Green chemistry on a BioMark qPCR apparatus (Fluidigm, San Francisco, CA, USA) following the manufacturer's instructions. For each cDNA sample, specific target amplification (STA) was performed with a pool of primers targeting all selected genes (preamplification of 14 cycles using the TaqMan PreAmp Master Mix, Applied Biosystems, Waltham, MA, USA) and following the manufacturer's instructions. Each qPCR was performed with $1 / 20$ STA dilution, in duplicate. Relative gene expression kinetics was created by a first normalization with four reference genes followed by a second normalization with non-infected (NI) macrophage cells. Values are expressed in fold changes $\left(2^{-\Delta \Delta C T}\right.$ method) compared to NI macrophage cells.

\section{Statistical Analysis}

Data were presented as the mean and SD (standard deviation). All graphs generated and the related statistical analysis were performed using GraphPad Prism. Statistical analysis was performed using ANOVA. Significance was reached with $p$ values $<0.05$. $P$-values were shown as ${ }^{\star} p<0.05$, ${ }^{* *} p<0.01$, ${ }^{* * *} p<0.001$, and ${ }^{* * * *} p<0.0001$.

\section{RESULTS}

\section{Leishmania Infection Drives HO-1 Expression}

We first assessed the ability of $L$. major parasites to induce the expression of HO-1. Balb/c BMdMs and/or Raw264.7 macrophages were infected with $L$. major promastigotes. 
The infected cells were harvested and the expressions of HO-1 messenger RNA (mRNA) and proteins were analyzed. Our results showed that promastigotes significantly induced the transcription and the protein expression of HO-1 (Figure 1).

\section{L. major Infection Leads to Activation of the Nrf2 Pathway}

Because the expressions of antioxidant genes are under the control of the Nrf2 transcription factor (TF), the activation of this master TF was investigated.

BMdMs from Balb/c mice or Raw264.7 cells were infected with $L$. major promastigotes for different times. The mRNA and protein were extracted for RT-PCR and Western blotting. The quantitative RTPCR (qRT-PCR) results showed a slight but significant increase in the expression of Nrf2 mRNA, which peaked at $3 \mathrm{~h}$ post-infection (hpi) (Figure 2A). The parasite-induced expression of the NRF2 protein was detected as early as $30 \mathrm{~min}$ pi, and the abundance peaked between 4 and 6 hpi before declining progressively (Figure 2B). These data suggested an upregulation of the expression of Nrf2 by the increased transcription of the Nrf2 gene leading to increased Nrf2 protein levels in infected cells. To confirm the activation of the Nrf2 pathway, we visualized the nuclear translocation of the Nrf2 protein. Subcellular fractionation showed Nrf2 accumulation in the nucleus following infection. Nrf2 was, during the first hour post-infection, predominantly localized in the cytoplasm. At $3 \mathrm{hpi}$, the Nrf2 protein started to be exported to the nucleus, and the nuclear localization of NRF2 was observed until 12 hpi (Figure 2C).

\section{PI3K/Akt and NRF2 Regulate the Expression of HO-1}

To determine the upstream regulators of HO-1, macrophages were infected or pretreated with a phosphatidylinositol 3-kinase (PI3K) inhibitor, wortmannin, and then infected by L. major promastigotes. Our results showed that wortmannin treatment decreased the parasite-induced phosphorylation of Akt (Figures 3A, C) and the expression of $\mathrm{HO}-1$ protein (Figures 3B, D), showing that PI3K/Akt activity is required to induce HO-1 expression during Leishmania infection.

To investigate the antioxidant function of Nrf2, Raw246.7 siNRF2 knockdown cells were generated. As expected, cells with disrupted Nrf2 did not express the silenced TF in response to LPS stimulation (Figure 3E). The effect of Nrf2 knockdown on the immunomodulatory expression of $\mathrm{HO}-1$ protein was investigated with Western blot. As shown in Figure 3F, the silencing of Nrf2 strongly reduced the expression of HO-1 in response to Leishmania infection, suggesting that, in response to Leishmania infection, the increased expression of $\mathrm{HO}-1$ is dependent on the Nrf2 TF.

\section{Activation of NRF2 and HO-1 Transcription by Leishmania Parasites Is Independent of the Genetic Background}

To determine whether Leishmania infection differentially regulates the mRNA expression of NRF2 and HO-1 in resistant and susceptible mice, $\mathrm{Balb} / \mathrm{c}$ and $\mathrm{C} 57 \mathrm{Bl} / 6 \mathrm{BMdMs}$ were infected with $L$. major promastigotes for different times. Infected cells were harvested and the expressions of antioxidant genes were analyzed using RT-PCR.

Our results showed that both Nrf2 and HO-1 mRNAs were significantly induced by the parasites in $\mathrm{C} 57 \mathrm{Bl} / 6 \mathrm{BMdMs}$ (Figure 4). The transcriptional kinetics of $\mathrm{Nrf} 2$ is very similar in BMdMs from both mouse strains, with slightly higher expression levels in $\mathrm{C} 57 \mathrm{Bl} / 6$ BMdMs. Regarding $\mathrm{HO}-1$, the transcription peaked earlier (3 hpi), lasted longer, and was at $12 \mathrm{~h}$ twice as important in $\mathrm{C} 57 \mathrm{Bl} / 6 \mathrm{BMdMs}$. Killed parasites
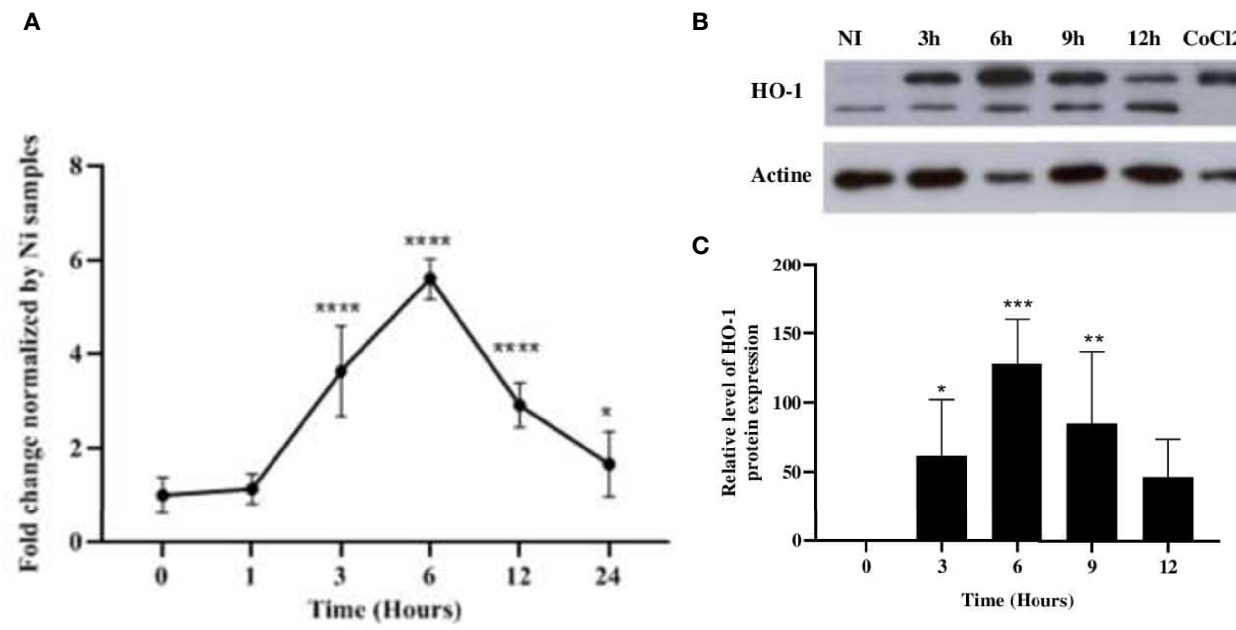

FIGURE 1 | Leishmania major induced the expression of HO-1 at both the mRNA and protein levels. Balb/c bone marrow-derived macrophages (BMdMs) and/or Raw264.7 cells were infected for the indicated time periods with L. major promastigotes. (A) The extracted mRNAs were used to perform RT-PCR. (B) The expression of heme oxygenase 1 (HO-1) was evaluated at the protein level by immunoblotting. $\mathrm{CoCl}_{2}$ stimulation $(250 \mathrm{ng} / \mathrm{ml})$ for $3 \mathrm{~h}$ was used as control. (C) Densitometric quantification of HO-1 protein levels using ImageJ. All data are expressed as the mean \pm SD from three independent experiments. Statistical significance was placed at $p<0.05$. ${ }^{*} p<0.05,{ }^{* *} p<0.01,{ }^{* \star *} p<0.001,{ }^{* \star * *} p<0.0001$ vs. non-stimulated cells (two-way ANOVA). 
A

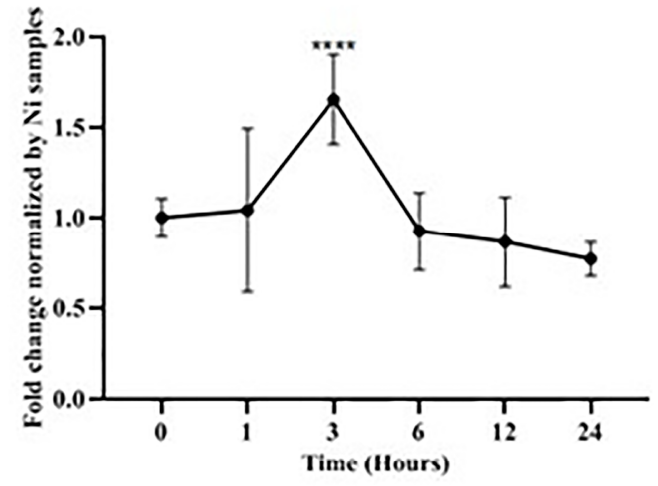

D

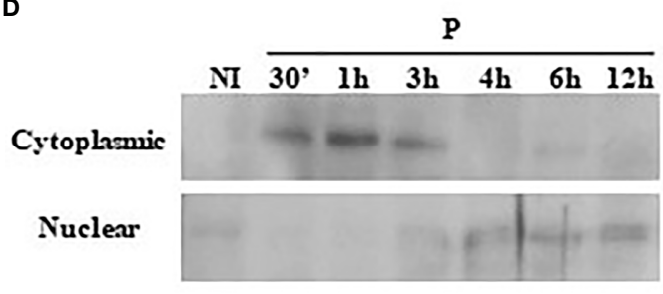

B

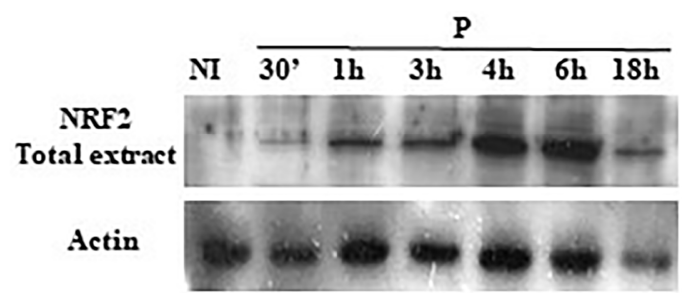

C

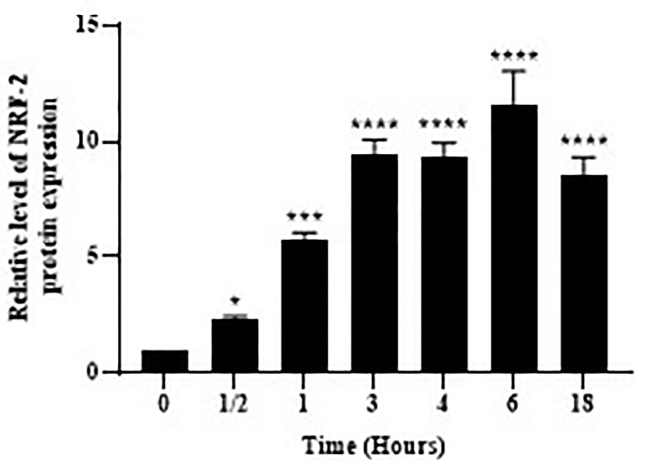

FIGURE 2 | Stimulation and nuclear accumulation of Nrf2 in response to Leishmania major infection. Balb/c bone marrow-derived macrophages (BMdMs) and/or Raw264.7 cells were infected for the indicated time period with L. major promastigotes. (A) The extracted mRNAs were used to perform RT-PCR. (B) Total Nrf2 expression was evaluated at the protein level by immunoblotting. (C) Densitometric quantification of the NRF2 protein levels using ImageJ. All data are expressed as the mean \pm SD from two independent experiments. ${ }^{*} p<0.05,{ }^{\star \star \star} p<0.001,{ }^{\star \star \star \star} p<0.0001$ vs. non-stimulated BMdMs (two-way ANOVA). (D) Total protein extracts were fractionated into cytoplasmic and nuclear extracts and immunoblots performed with an anti-Nrf2 antibody. ERK1/2 and PARP were used to confirm the efficacy of cytosol and nuclear fractionation.

induced NRF2 and HO-1 to nearly the same extent as did the live parasites in BMdMs from both mouse strains.

\section{The Impact of Leishmania Infection on the Expressions of Several NRF2 Antioxidant Genes}

We investigated the transcription of different NRF2-regulated antioxidant genes in the infected macrophages. Our results showed that, in Balb/c BMdMs, Leishmania increased the transcription of the cysteine/glutamate exchange transporter (Slc7a11) regulating the cysteine influx and that of the catalytic (GCLc) and modifier (GCLm) subunits of the $\gamma$-glutamylcysteine ligase, the rate-limiting enzyme regulating the synthesis of glutathione (Figure 5). The parasites also increased the transcription of glutathione reductase (Gsr), CD36, and catalase (CAT). The transcription of almost all of the antioxidant genes peaked at $6 \mathrm{hpi}$, except for that of Gsr, which peaked earlier (3 hpi). Similar results were obtained in C57Bl/6 BMdMs, except for Slc7a11 and Gsr (Figure 6). Indeed, Slc7a11 mRNA was fivefold and Gsr mRNA was 20-fold higher in $\mathrm{C} 57 \mathrm{Bl} / 6 \mathrm{BMdMs}$. Moreover, when compared to that induced by inactivated parasites, Gsr was not only transcribed to a lesser extent but was also repressed at all time points by live parasites in $\mathrm{Balb} / \mathrm{c}$-derived macrophages. In fact, the transcription of almost all of the selected genes was actively repressed by the live parasite; this repression was observed at all time points for Gsr, CD36, and CAT and at the later times for the other genes.

\section{NRF2 Silencing Promotes Leishmania Persistence and Proliferation Inside Macrophages}

Intracellular pathogens such as Leishmania use macrophages as a reservoir for dissemination. To test the ability of Nrf2 expression in controlling parasite load and multiplication, Ds-Red promastigotes were used to investigate the intracellular load of Leishmania. NT-pLKO raw cells and deficient NRF2 cells were infected with Ds-Red promastigotes for $72 \mathrm{~h}$ and the parasite load was analyzed by flow cytometry. Our experiments showed that Nrf2 loss in infected macrophages increased the ability of amastigotes to multiply inside the macrophages; parasite fluorescence emission is significantly more important in NRF2 knockdown cells (Figure 7).

\section{DISCUSSION}

Macrophage-Leishmania interaction and the response of macrophages to infection direct the outcome of the disease. 


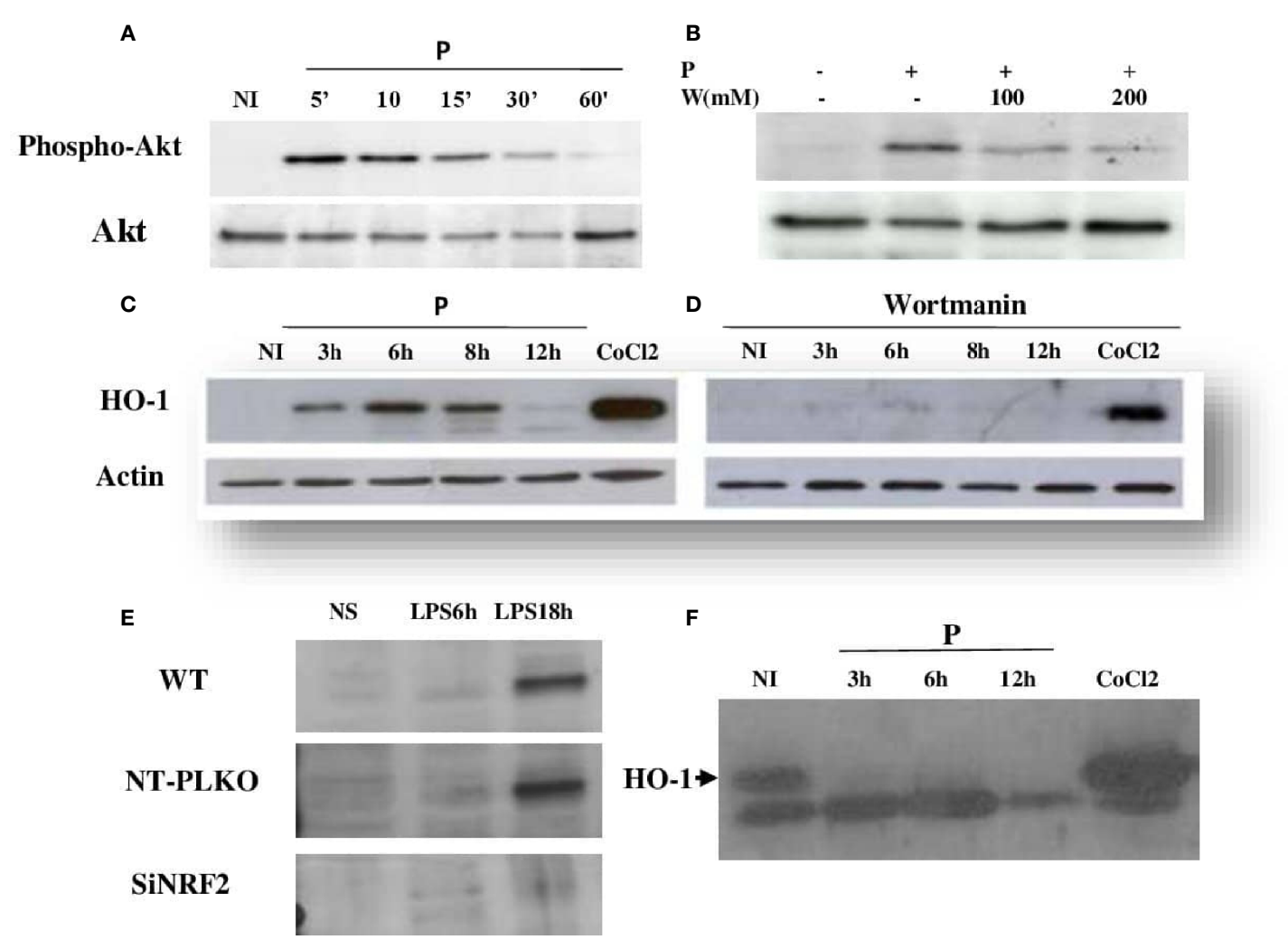

FIGURE 3 | Leishmania major induced HO-1 expression through PI3K/Akt and the Nrf2 transcription factor. (A, C) Raw264.7 cells were infected for the indicated time periods with L. major promastigotes and the expressions of Akt and $\mathrm{HO}-1$ assessed using Western blot. $\mathrm{CoCl}_{2}$ stimulation for $3 \mathrm{~h}$ (250 $\mathrm{ng} / \mathrm{ml}$ ) was used as the control. (B, D) Macrophages were pre-incubated with wortmannin (200 mM) for $3 \mathrm{~h}$, followed by infection with $\mathrm{L}$. major promastigotes for different times. Akt phosphorylation and HO-1 expression were determined by immunoblotting. (E) Raw264.7 cells were transfected (24 h) with either Nrf2 (Si) or control siRNA (NTpLKO), followed by infection with lipopolysaccharide (LPS) for $18 \mathrm{~h}$, lysed, and examined by Western blot for Nrf2 expression. (F) NRF2 ${ }^{-/-}$cells were infected for different times with L. major promastigotes, lysed, and examined by Western blot for HO-1 expression.

Parasite infection activates microbicidal mechanisms such as oxidative response that help protect the host against the invaders, but can be harmful for the cells. The Nrf2 signaling pathway leads to the activation of all defense genes that aim to protect the cells from the oxidative response switched to face the infection. The Nrf2 TF plays a key role in the maintenance of intracellular redox homeostasis and the regulation of inflammation. Among the antioxidative stress genes whose expressions are regulated by NRF2, HO-1, encoded by the Hmoxl gene, has received considerable attention. HO-1 is responsible for heme detoxification and, apart from ROS neutralization, has a potent anti-inflammatory effect and plays a critical role in iron homeostasis (Vijayan et al., 2018). The inducible HO-1 isoform is induced in response to Helicobacter pylori infection (Gobert et al., 2014), required for protection against Toxoplasma gondii (Araujo et al., 2013), and critically contributes to host resistance to Mycobacterium infections in mice (Silva-Gomes et al., 2013). It is also activated in response to protozoan parasites, such as Trypanosoma brucei (Campbell et al., 2019) and Trypanosoma cruzi (Paiva et al., 2012). Our results indicated that L. major also increased the expression of HO-1 both at the mRNA and protein levels (Figure 1). This result is consistent with previous studies showing an increased expression of HO-1 in macrophages in response to different species of Leishmania in either of its two forms (Pham et al., 2005; Luz et al., 2012; de Menezes et al., 2019; Saha et al., 2019).

HO- 1 activation is dependent on the PI3K/Akt and Nrf2 signaling pathways. Indeed, we showed that L. major, as previously reported, induced the PI3K/Akt pathway (Ruhland et al., 2007) and the activation of the Nrf2 TF and its translocation to the nucleus (de Menezes et al., 2019). Wortmannin treatment and NRF2 silencing showed that these two pathways were both involved in the regulation of HO-1 expression (Figure 2).

HO-1 expression orchestrated by the activation of the Nrf2 promoter has been associated with parasite survival and persistence, as the BMdMs from $\mathrm{Hmox}^{-/-} \mathrm{Balb} / \mathrm{c}$ mice presented a significantly reduced Leishmania chagasi parasite burden (Luz et al., 2012). In contrast, for other different pathogens including Trypanosomatidae, HO-1 seems to protect the host against the invaders. Indeed, inhibition of the activity of HO-1 increased T. cruzi parasitemia (Paiva et al., 2012). Similarly, the silencing of HO-1 in H. pyloriinfected macrophages downregulated M1 polarization and favored H. pylori survival (Gobert et al., 2014). HO-1-deficient mice developed higher pathogen loads and were more susceptible to intravenous Mycobacterium avium infection, suggesting that $\mathrm{HO}-1$ 
Balb/C
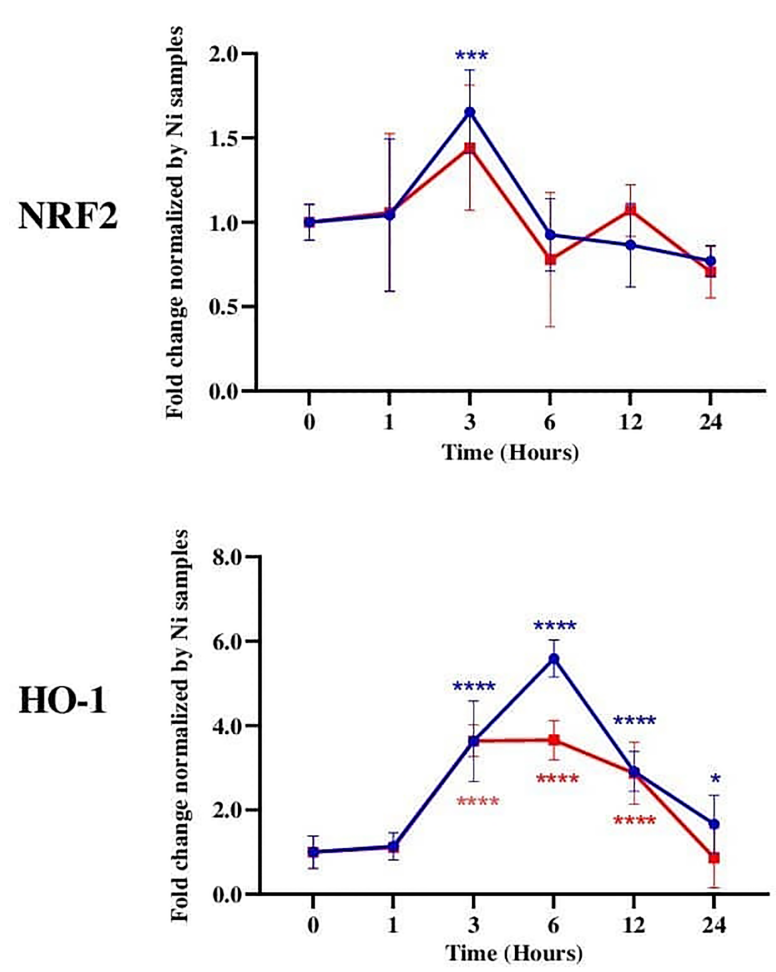

C57BI/6
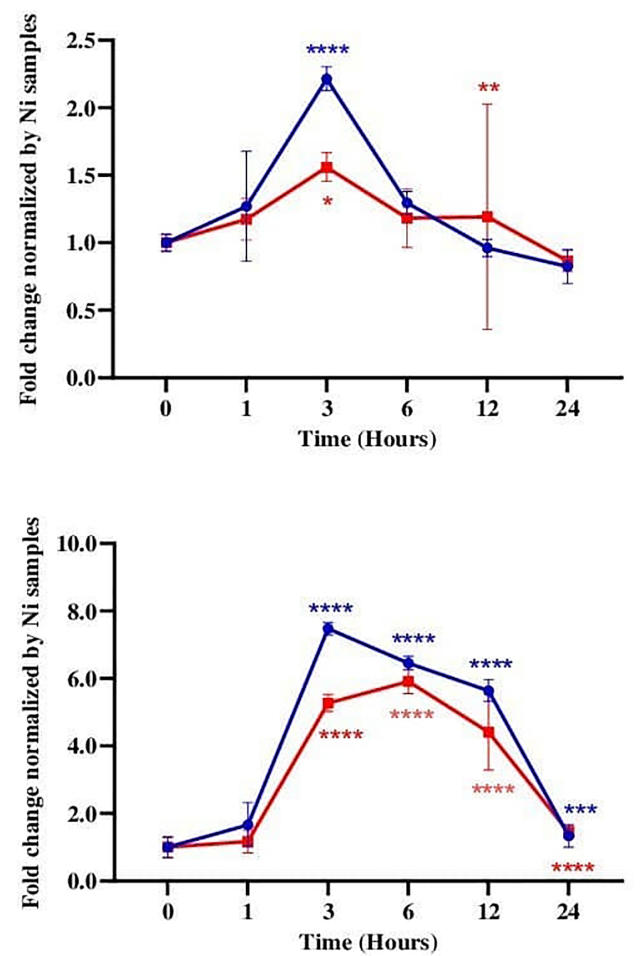

FIGURE 4 | NRF2 and HO-1 mRNAs were induced in Leishmania-susceptible and Leishmania-resistant bone marrow-derived macrophages (BMdMs). Balb/c and C57Bl6 BMdMs were infected by live parasites $(P)$ (blue filled circle) or heat-inactivated $(K p)$ (red filled square) $L$. major promastigotes for different times. RT-PCR targeting Nrf2 and HO-1 was performed. The graphs show fold change results expressed as the mean \pm SD from three independent experiments. ${ }^{*} p<0.05$, ${ }^{* \star} p<0.01,{ }^{* \star *} p<0.001,{ }^{\star * \star *} p<0.0001$ vs. non-stimulated BMdMs (two-way ANOVA).

expression in macrophages is strictly required for protection against mycobacterial infection in mice (Silva-Gomes et al., 2013).

The host genetic background plays a key role in the development of leishmaniasis and has a strong impact on the severity and the final outcome of the disease. C57BL/6 and $\mathrm{BALB} / \mathrm{c}$ mice, the prototypical Th1- and Th2-type mouse strains, are respectively resistant and susceptible to Leishmania parasites. The innate immune response of macrophages, different between these mouse strains, certainly affects the development of the adaptive immunity of Th1 and Th2 (Watanabe et al., 2004). We tried first to determine whether the expressions of NRF2 and HO-1 are different in these two mouse strains showing contrasting behaviors in response to Leishmania infection. Our results revealed that the transcriptions of NRF2 and HO-1 were not differentially regulated and were similarly induced in BMdMs issued from either $\mathrm{Balb} / \mathrm{C}$ or $\mathrm{C} 57 \mathrm{Bl} / 6$ (Figure 4). Additionally, the transcription of $\mathrm{HO}-1$, which can drive the phenotypic shift to M2 macrophages (Naito et al., 2014) and favor parasite survival (Pham et al., 2005; Luz et al., 2012), peaked earlier, lasted longer, and was not repressed by the parasites in BMdMs from resistant mice. However, HO-1 has, in addition, significant anti-inflammatory effects (Vijayan et al.,
2018; Costa et al., 2020) and induces protective enzymes sequestering iron ions (Loboda et al., 2016).

Besides HO-1, GSH systems, one of the two major thioldependent antioxidant mechanisms in cells, are also activated by L. major infection. Our results also point out that initial contact with Leishmania was necessary and sufficient to promote the macrophage activation of the Nrf2 pathway. This pathway is therefore likely triggered by the stimulation of the receptors implicated in the recognition of the parasites, as heat-inactivated parasites also have the ability to induce the transcription of both NRF2 and its target genes.

All of the selected target genes were induced in both Balb/c and C57B16 BMdMs. However, the transcriptions of the cysteine transporter (Slc7a11) and of Gsr were differentially regulated between the macrophages from the two mouse strains. Indeed, these genes were more highly transcribed in C57Bl6 BMdMs. This increased transcription of Slc7a11, which is required to maintain the intracellular level of GSH and that of Gsr, involved in the recycling of oxidized glutathione, may result in glutathione accumulation, which has been reported to play a role in the resistance to Leishmania infection. Indeed, the depletion of glutathione has been shown to significantly increase the 


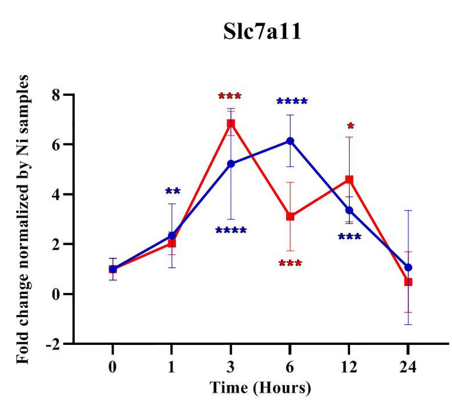

Gsr

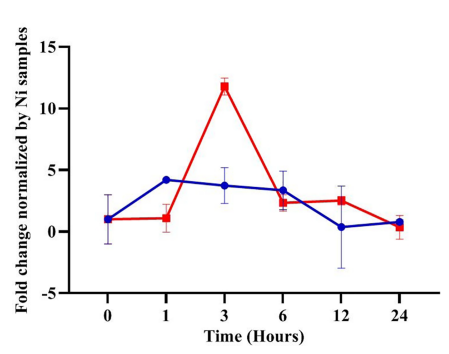

Gclc

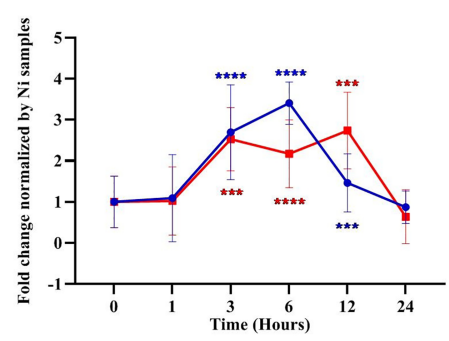

CAT

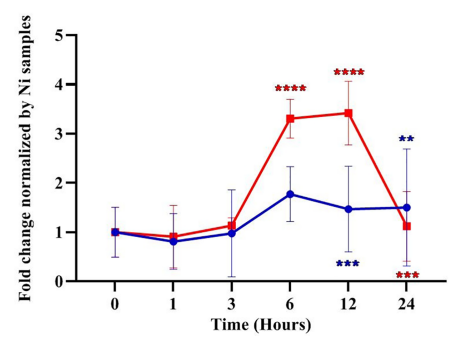

Gclm

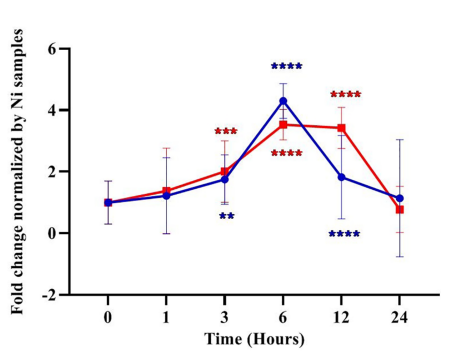

CD36

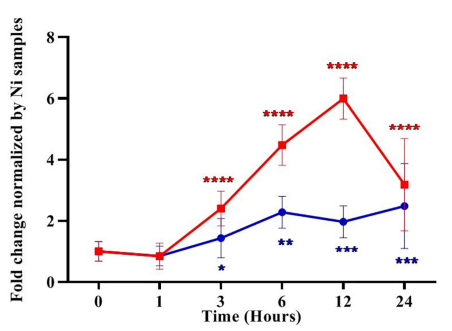

FIGURE 5 | Transcription of different antioxidant genes was repressed by parasites. Balb/c bone marrow-derived macrophages (BMdMs) were infected with live parasites (P) (blue filled circle or heat-inactivated (Kp) (red filled square) L. major promastigotes for different times. RT-PCR targeting Slc7a11, GCLc, GCLm, Gsr, CAT, and CD36 was performed. The graphs show fold change results expressed as the mean \pm SD from three independent experiments. ${ }^{*} p<0.05$, ${ }^{* *} p<0.01$, ${ }^{\star \star \star} p<0.001,{ }^{\star \star \star \star} p<0.0001$ vs. non-stimulated BMdMs (two-way ANOVA).

parasite load in the footpads of C57BL/6 mice infected with $L$. major (Cruz et al., 2008). Moreover, a close correlation was observed between the levels of intracellular soluble GSH and the secretion of nitrite (Buchmüller-Rouiller et al., 1995). In fact, a reduced cellular environment is important for several cellular functions, such as immune response and macrophage polarization (Fraternale et al., 2017). Thus, in contrast to HO1, the transcription of Slc7a11 and Gsr, belonging to the glutathione system, was differentially regulated in BMdMs from resistant and susceptible mice, suggesting that glutathione may play an important role in the elimination of the parasite.

The second most striking observation is that, although the transcription of all the targeted antioxidant genes was induced by the infection and the triggering of the receptors, it was actively repressed by the parasite. This was particularly true for CD36 and CAT, a peroxide-scavenging enzyme, and for Gsr in BMdMs from susceptible mice.

CD36 represents an important part of the innate immune defense and may act as a pattern recognition receptor, in particular against bacterial pathogens (Areschoug and Gordon, 2009). CD36 is also important for host resistance to infection, and its deficiency significantly reduces mycobacteria burden (Hawkes et al., 2010), whereas its induction helps control severe malaria through parasite clearance (Olagnier et al., 2011). Increased transcription of CD36 has been reported for Leishmania infantum (Gatto et al., 2020) and Leishmania amazonensis (Okuda et al., 2016). However, while $\mathrm{CD}^{-1-}$ macrophages are infected but do not support L. amazonensis proliferation, L. major amastigotes do not recruit CD36 and proliferate normally in $\mathrm{CD}^{-1-}$ macrophages (Okuda et al., 2016). This is consistent with our finding that, despite their ability to induce NRF2 expression, L. major promastigotes actively limited CD36 transcription. In a different model, CD36 has been implicated in the regulation of ROS. Indeed, in murine vascular smooth muscle cells (VSMCs), while ROS induced the activation of the NRF2 target genes to limit oxidant stress, it also led to the generation of specific CD36 ligands, such as MP and oxLDL, allowing the scavenger receptor to phosphorylate NRF2 and, thus, to induce its exit from the nucleus and degradation (Li et al., 2010).

Thus, except for HO-1, the transcription of all the Nrf2 target genes tested was repressed, if not at all time points at least at the later ones, suggesting that these enzymes may play a role in limiting parasite survival and/or growth.

Our result showing that the silencing of the Nrf2 TF induced an increase in the parasite load is therefore in agreement with the parasite repressing the expressions of NRF2 target genes.

The role of NRF2 has also been explored, and transcriptomic and proteomic analyses highlighted the importance of $\mathrm{Nrf} 2$ signaling in cutaneous leishmaniasis (Vivarini et al., 2017; de Menezes et al., 2019). Indeed, the activation of NRF2 induced by L. amazonensis and Leishmania braziliensis enhanced the intracellular pathogen survival and disease progression (Vivarini et al., 2017). L. amazonensis-infected bone marrow macrophages exhibited increased expressions of NRF2 and antioxidant HO-1, which, together with higher levels of holotransferrin (holoTf) in parasitophorous vacuoles, contributed to the persistence of 


\section{Balb/C}

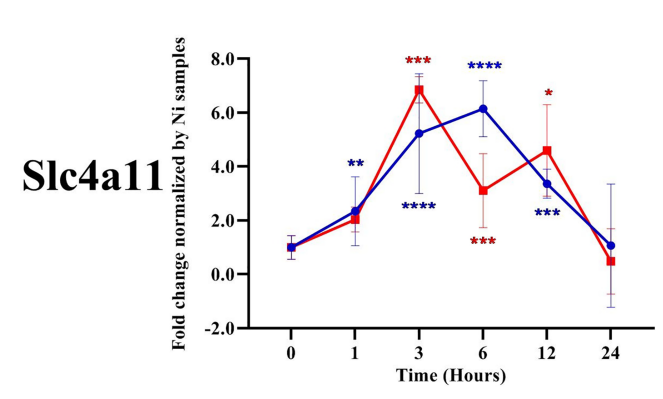

Gsr

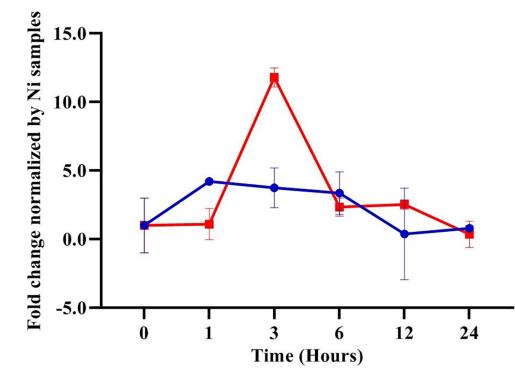

C57BI/6
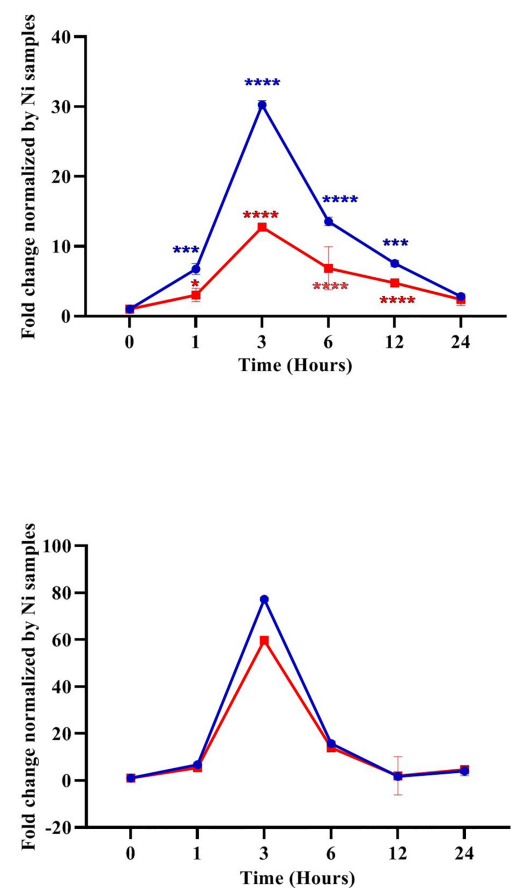

FIGURE 6 | Slc7a11 and Gsr were differentially regulated by Leishmania parasites in Balb/c and C57Bl6 bone marrow-derived macrophages (BMdMs). Balb/c and C57Bl6 BMdMs were infected by live parasites $(P)$ (b/ue filled circle) or heat-inactivated $(K p)$ (red filled square) $L$. major promastigotes for different times. RT-PCR targeting Slc7a11 and Gsr was performed. The graphs show fold change results expressed as the mean \pm SD from three independent experiments. ${ }^{*} p<0.05$, ${ }^{\star \star} p<0.01,{ }^{\star \star \star} p<0.001,{ }^{\star \star \star \star} p<0.0001$ vs. non-stimulated BMdMs (two-way ANOVA).

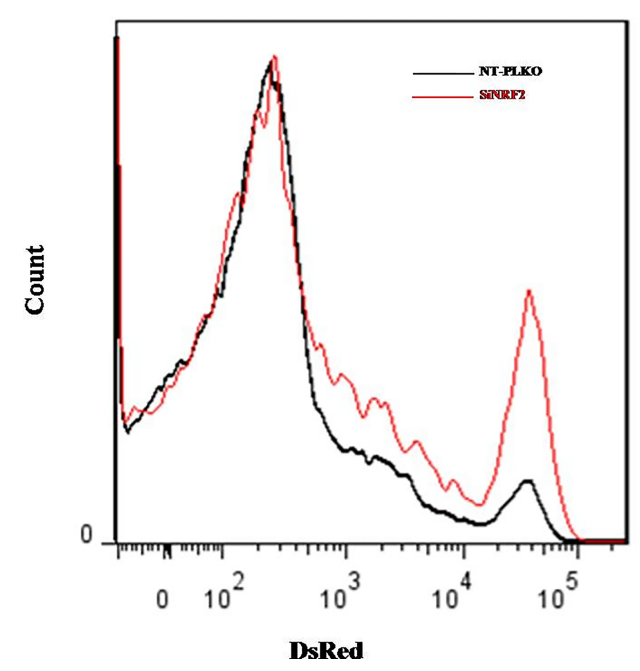

FIGURE 7 | Silencing of NRF2 promoted parasite survival. NT-pLKO or si-NRF2 Raw264.7 cells were infected with Ds-Red promastigotes. Macrophages (MФs) parasitized with Ds-Red parasites were analyzed $72 \mathrm{~h}$ post-infection by flow cytometry. The percentages of Ds-Red fluorescence corresponding to the multiplication of amastigotes in parasitized MФs were compared in NT-pLKO and si-NRF2-infected macrophages.
L. amazonensis infection (de Menezes et al., 2019). In our study, however, the silencing of NRF2 induced the persistence and multiplication of $L$. major parasites. This is in accordance with earlier results showing that treatment of L. major-infected BALB/c mice with $\mathrm{N}$-acetyl-l-cysteine (NAC), a glutathione precursor and an NRF2 inducer, reduced the parasitism in their footpads (RochaVieira et al., 2003).

We show in the present study that, while the master regulator of antioxidant response genes, Nrf2, is strongly induced by macrophage-Leishmania contact, the parasite represses the transcription of antioxidant genes and that the inactivation of the Nrf2 TF favors parasite survival and multiplication. Moreover, NRF2 target genes appear to play contrasting roles in Leishmania-infected macrophages, with HO-1 enabling parasite survival and enzymes of the GSH system, which are differentially regulated between BMdMs from resistant and susceptible mice, promoting parasite removal.

\section{DATA AVAILABILITY STATEMENT}

The original contributions presented in the study are included in the article/supplementary material. Further inquiries can be directed to the corresponding author. 


\section{ETHICS STATEMENT}

The animal study was reviewed and approved by the Ethics Committee of Institute Pasteur of Tunis, with ethics approval no. 1204.

\section{AUTHOR CONTRIBUTIONS}

$\mathrm{HB}, \mathrm{SR}, \mathrm{CH}$, and $\mathrm{CB}$ carried out the experiments. MB helped with the flow cytometry experiments. DP and BT performed the

\section{REFERENCES}

Araujo, E. C. B., Barbosa, B. F., Coutinho, L. B., Barenco, P. V. C., Sousa, L. A., Milanezi, C. M., et al. (2013). Heme Oxygenase-1 Activity Is Involved in the Control of Toxoplasma Gondii Infection in the Lung of BALB/c and C57BL/6 and in the Small Intestine of C57BL/6 Mice. Vet. Res. 44, 89. doi: 10.1186/12979716-44-89

Areschoug, T., and Gordon, S. (2009). Scavenger Receptors: Role in Innate Immunity and Microbial Pathogenesis. Cell Microbiol. 11, 1160-1169. doi: 10.1111/j.1462-5822.2009.01326.x

Baird, L., and Yamamoto, M. (2020). The Molecular Mechanisms Regulating the KEAP1-NRF2 Pathway. Mol. Cell Biol. 40, e00099-e00020. doi: 10.1128/ MCB.00099-20

Buchmüller-Rouiller, Y., Corrandin, S. B., Smith, J., Schneider, P., Ransijn, A., Jongeneel, C. V., et al. (1995). Role of Glutathione in Macrophage Activation: Effect of Cellular Glutathione Depletion on Nitrite Production and Leishmanicidal Activity. Cell Immunol. 164, 73-80. doi: 10.1006/ cimm.1995.1144

Campbell, N. K., Williams, D. G., Fitzgerald, H. K., Barry, P. J., Cunningham, C. C., Nolan, D. P., et al. (2019). Trypanosoma Brucei Secreted Aromatic Ketoacids Activate the Nrf2/HO-1 Pathway and Suppress Pro-Inflammatory Responses in Primary Murine Glia and Macrophages. Front. Immunol. 10, 2137. doi: 10.3389/fimmu.2019.02137

Conceição-Silva, F., and Morgado, F. N. (2019). Leishmania Spp-Host Interaction: There Is Always an Onset, But Is There an End? Front. Cell Infect. Microbiol. 9, 330. doi: 10.3389/fcimb.2019.00330

Costa, D. L., Amaral, E. P., Andrade, B. B., and Sher, A. (2020). Modulation of Inflammation and Immune Responses by Heme Oxygenase-1: Implications for Infection With Intracellular Pathogens. Antioxid. (Basel) 9, E1205. doi: 10.3390/antiox9121205

Cruz, K. K., Fonseca, S. G., Monteiro, M. C., Silva, O. S., Andrade, V. M., Cunha, F. Q., et al. (2008). The Influence of Glutathione Modulators on the Course of Leishmania Major Infection in Susceptible and Resistant Mice. Parasit. Immunol. 30, 171-174. doi: 10.1111/j.1365-3024.2007.01014.x

de Menezes, J. P. B., Khouri, R., Oliveira, C. V. S., Petersen, A. L., de, O. A., de Almeida, T. F., et al. (2019). Proteomic Analysis Reveals a Predominant NFE2L2 (NRF2) Signature in Canonical Pathway and Upstream Regulator Analysis of Leishmania-Infected Macrophages. Front. Immunol. 10, 1362. doi: 10.3389/fimmu.2019.01362

Fraternale, A., Brundu, S., and Magnani, M. (2017). Glutathione and Glutathione Derivatives in Immunotherapy. Biol. Chem. 398, 261-275. doi: 10.1515/hsz2016-0202

Gatto, M., Borim, P. A., Wolf, I. R., Fukuta da Cruz, T., Ferreira Mota, G. A., Marques Braz, A. M., et al. (2020). Transcriptional Analysis of THP-1 Cells Infected With Leishmania Infantum Indicates No Activation of the Inflammasome Platform. PloS Negl. Trop. Dis. 14, e0007949. doi: 10.1371/journal.pntd.0007949

Gobert, A. P., Verriere, T., Asim, M., Barry, D. P., Piazuelo, M. B., de Sablet, T., et al. (2014). Heme Oxygenase-1 Dysregulates Macrophage Polarization and the Immune Response to Helicobacter Pylori. J. Immunol. 193, 3013-3022. doi: 10.4049/jimmunol.1401075

Hawkes, M., Li, X., Crockett, M., Diassiti, A., Finney, C., Min-Oo, G., et al. (2010). CD36 Deficiency Attenuates Experimental Mycobacterial Infection. BMC Infect. Dis. 10, 299. doi: 10.1186/1471-2334-10-299
qRT-PCR. IR supervised and contributed to the interpretation of the results. LG-T conceived and planned the experiments and wrote the manuscript. All authors contributed to the article and approved the submitted version.

\section{FUNDING}

This study was funded by the Tunisian Ministry of Higher Education.

Itoh, K., Ishii, T., Wakabayashi, N., and Yamamoto, M. (1999). Regulatory Mechanisms of Cellular Response to Oxidative Stress. Free Radic. Res. 31, 319-324. doi: 10.1080/10715769900300881

Li, W., Febbraio, M., Reddy, S. P., Yu, D.-Y., Yamamoto, M., and Silverstein, R. L. (2010). CD36 Participates in a Signaling Pathway That Regulates ROS Formation in Murine VSMCs. J. Clin. Invest. 120, 3996-4006. doi: 10.1172 JCI42823

Loboda, A., Damulewicz, M., Pyza, E., Jozkowicz, A., and Dulak, J. (2016). Role of Nrf2/HO-1 System in Development, Oxidative Stress Response and Diseases: An Evolutionarily Conserved Mechanism. Cell Mol. Life Sci. 73, 3221-3247. doi: 10.1007/s00018-016-2223-0

Luz, N. F., Andrade, B. B., Feijó, D. F., Araújo-Santos, T., Carvalho, G. Q., Andrade, D., et al. (2012). Heme Oxygenase-1 Promotes the Persistence of Leishmania Chagasi Infection. J. Immunol. 188, 4460-4467. doi: 10.4049/ jimmunol.1103072

Naito, Y., Takagi, T., and Higashimura, Y. (2014). Heme Oxygenase-1 and AntiInflammatory M2 Macrophages. Arch. Biochem. Biophys. 564, 83-88. doi: 10.1016/j.abb.2014.09.005

Nguyen, T., Sherratt, P. J., and Pickett, C. B. (2003). Regulatory Mechanisms Controlling Gene Expression Mediated by the Antioxidant Response Element. Annu. Rev. Pharmacol. Toxicol. 43, 233-260. doi: 10.1146/ annurev.pharmtox.43.100901.140229

Okuda, K., Tong, M., Dempsey, B., Moore, K. J., Gazzinelli, R. T., and Silverman, N. (2016). Leishmania Amazonensis Engages CD36 to Drive Parasitophorous Vacuole Maturation. PloS Pathog. 12, e1005669. doi: 10.1371/ journal.ppat.1005669

Olagnier, D., Lavergne, R.-A., Meunier, E., Lefèvre, L., Dardenne, C., Aubouy, A., et al. (2011). Nrf2, a Ppary Alternative Pathway to Promote CD36 Expression on Inflammatory Macrophages: Implication for Malaria. PloS Pathog. 7 e1002254. doi: 10.1371/journal.ppat.1002254

Paiva, C. N., Feijó, D. F., Dutra, F. F., Carneiro, V. C., Freitas, G. B., Alves, L. S. et al. (2012). Oxidative Stress Fuels Trypanosoma Cruzi Infection in Mice. J. Clin. Invest. 122, 2531-2542. doi: 10.1172/JCI58525

Pham, N.-K., Mouriz, J., and Kima, P. E. (2005). Leishmania Pifanoi Amastigotes Avoid Macrophage Production of Superoxide by Inducing Heme Degradation. Infect. Immun. 73, 8322-8333. doi: 10.1128/IAI.73.12.8322-8333.2005

Rabhi, S., Rabhi, I., Trentin, B., Piquemal, D., Regnault, B., Goyard, S., et al. (2016). Lipid Droplet Formation, Their Localization and Dynamics During Leishmania Major Macrophage Infection. PloS One 11, e0148640. doi: 10.1371/journal.pone.0148640

Rocha-Vieira, E., Ferreira, E., Vianna, P., De Faria, D. R., Gaze, S. T., Dutra, W. O., et al. (2003). Histopathological Outcome of Leishmania Major-Infected BALB/c Mice Is Improved by Oral Treatment With N-Acetyl-L-Cysteine. Immunology 108, 401-408. doi: 10.1046/j.1365-2567.2003.01582.x

Ruhland, A., Leal, N., and Kima, P. E. (2007). Leishmania Promastigotes Activate PI3K/Akt Signalling to Confer Host Cell Resistance to Apoptosis. Cell Microbiol. 9, 84-96. doi: 10.1111/j.1462-5822.2006.00769.x

Saha, S., Basu, M., Guin, S., Gupta, P., Mitterstiller, A.-M., Weiss, G., et al. (2019). Leishmania Donovani Exploits Macrophage Heme Oxygenase-1 To Neutralize Oxidative Burst and TLR Signaling-Dependent Host Defense. J. Immunol. 202, 827-840. doi: 10.4049/jimmunol.1800958

Silva-Gomes, S., Appelberg, R., Larsen, R., Soares, M. P., and Gomes, M. S. (2013). Heme Catabolism by Heme Oxygenase-1 Confers Host Resistance to 
Mycobacterium Infection. Infect. Immun. 81, 2536-2545. doi: 10.1128/ IAI.00251-13

Sykiotis, G. P., and Bohmann, D. (2010). Stress-Activated Cap'n'collar Transcription Factors in Aging and Human Disease. Sci. Signal. 3, re3. doi: 10.1126/scisignal.3112re3

Tonelli, C., Chio, I. I. C., and Tuveson, D. A. (2018). Transcriptional Regulation by Nrf2. Antioxid. Redox. Signal. 29, 1727-1745. doi: 10.1089/ars.2017.7342

Vijayan, V., Wagener, F. A. D. T. G., and Immenschuh, S. (2018). The Macrophage Heme-Heme Oxygenase-1 System and Its Role in Inflammation. Biochem. Pharmacol. 153, 159-167. doi: 10.1016/j.bcp.2018.02.010

Vivarini, Á., de, C., Calegari-Silva, T. C., Saliba, A. M., Boaventura, V. S., FrançaCosta, J., et al. (2017). Systems Approach Reveals Nuclear Factor Erythroid 2Related Factor 2/Protein Kinase R Crosstalk in Human Cutaneous Leishmaniasis. Front. Immunol. 8, 1127. doi: 10.3389/fimmu.2017.01127

Watanabe, H., Numata, K., Ito, T., Takagi, K., and Matsukawa, A. (2004). Innate Immune Response in Th1- and Th2-Dominant Mouse Strains. Shock 22, 460466. doi: 10.1097/01.shk.0000142249.08135.e9
Conflict of Interest: The authors declare that the research was conducted in the absence of any commercial or financial relationships that could be construed as a potential conflict of interest.

Publisher's Note: All claims expressed in this article are solely those of the authors and do not necessarily represent those of their affiliated organizations, or those of the publisher, the editors and the reviewers. Any product that may be evaluated in this article, or claim that may be made by its manufacturer, is not guaranteed or endorsed by the publisher.

Copyright (C) 2021 Bichiou, Rabhi, Ben Hamda, Bouabid, Belghith, Piquemal, Trentin, Rabhi and Guizani-Tabbane. This is an open-access article distributed under the terms of the Creative Commons Attribution License (CC BY). The use, distribution or reproduction in other forums is permitted, provided the original author(s) and the copyright owner(s) are credited and that the original publication in this journal is cited, in accordance with accepted academic practice. No use, distribution or reproduction is permitted which does not comply with these terms. 